\title{
A Novel Multiple Access Protocol with QoS Support for Mobile Ad Hoc Networks
}

\author{
Dapeng Wang, Kai Liu, Lianzhen Cheng, and Yan Zhang \\ School of Electronics and Information Engineering, \\ Beijing University of Aeronautics and Astronautics, 100083 Beijing, China \\ bjwdp@sina.com, liuk@buaa.edu.cn, clz_tea@sina.com, \\ hiyanzy@yahoo.com.cn
}

\begin{abstract}
Based on the concept of random contention and collision resolution, a QoS-based multiple access (QMA) protocol for ad hoc networks is proposed to support multimedia service and multi-hop architecture. In this paper, the traffic is divided into two groups with different QoS requirements, namely realtime traffic and non real-time traffic. According to the protocol, nodes with real-time traffic have higher priority to access channel than those with non realtime traffic by broadcasting forecast bursts (FB) in earlier contention slots. Meanwhile, real-time traffic is scheduled according to its delay and the earliest deadline first (EDF) principle. Through simulations, it is shown that the QMA protocol outperforms the Carrier Sensing Multiple Access with Collision Avoidance (CSMA/CA) protocol in terms of throughput, message discard rate and average packet delay, and the QMA protocol can provide differentiated QoS guarantees for traffic in multi-hop networks.
\end{abstract}

Keywords: mobile ad hoc network, multiple access protocol, QoS, collision resolution, forecast burst.

\section{Introduction}

A mobile ad hoc network consists of a collection of mobile nodes without a fixed infrastructure, and it aims to set up and maintain a network for the nodes to communicate directly with each other. Medium access control (MAC) protocols are used for multiple nodes to share scarce bandwidth in an orderly and efficient manner.

Currently, there are many MAC protocols proposed for ad hoc networks, which can be classified into two categories: random access and on-demand assignment. In random access protocols, a node with data packets usually senses shared channel for a certain duration before packet transmission. If the channel is busy, the node waits for a random period and tries again at a later time. If the channel is idle, it makes an attempt to access the channel. A collision resolution mechanism is used to solve possible packet collisions in an orderly manner. Typical random access protocol is CSMA/CA coupled with request to send/clear to send (RTS/CTS) handshake in the distribution coordination function (DCF) of IEEE 802.11 MAC protocol [1]. Compared with the CSMA/CA without handshake, it decreases transmission collisions from the transmission time of long data packet to that of RTS mini-packet 
mostly due to hidden nodes, which are defined as nodes that are outside the communication range of the transmitter but inside the communication range of the receiver. However, its throughput still decreases drastically due to the increase of packet collisions when the total offered load is heavy [2, 3]. On the other hand, the on-demand assignment protocols generally use a central control point to assign channel resources for its neighbor nodes through reservation or polling mechanisms, such as point coordination function (PCF) of IEEE 802.11 protocol [1] and distributed packet reservation multiple access (D-PRMA) protocol [4]. However, their overhead and packet delay will increase when a large number of nodes have no packets to transmit.

To support multimedia traffic in fully distributed wireless ad hoc networks, quality of service (QoS) support in MAC layer is a very challenging issue. Generally, the existing QoS protocols achieve service differentiation by assigning different classes of traffic with different contention related parameters. For instance, enhanced distributed coordination function (EDCF) of IEEE 802.11e protocol [5] provides 4 access categories (AC) and 8 priorities, which are supported by assigning different contention window sizes and interframe spaces. However, the delay of high-priority packets is not bounded under heavy load in multi-hop networks, because high-priority packets may be blocked by alternate transmissions of low-priority packets within 2hop range, and unbounded delay for initiating an RTS/CTS dialogue cannot guarantee the delay of all the real-time packets. To solve these problems, many improved protocols based on IEEE 802.11 series have been presented. Yang proposes an algorithm in [6] which adapts initial back-off window size to guarantee QoS requirements in the IEEE 802.11 one-hop networks. QPART [7] uses contention window adaptation parameters based on service differentiation to provide QoS-aware scheduling for multi-hop ad hoc networks, and ensures that the resource requirements of admitted real-time traffic are smaller than the network capacity. In [8] Collision Avoidance Interval (CAI) is defined instead of Network Allocation Vector (NAV) used in IEEE 802.11, because NAV is too short for a collision to be prevented in the carrier sensing zone. Besides IEEE 802.11 and its improved protocols, some new competition schemes have been proposed. In Hiperlan protocol [9], nodes broadcast bursts to contend in different slots according to the priority and delay of packets. According to Priority Binary Countdown (PBC) scheme [10], each node selects an appropriate competition number $(\mathrm{CN})$ as signals to contend, which is composed of priority number part, random number part and ID number part. In each bit-slot of $\mathrm{CN}$, a node whose $\mathrm{CN}$ value is 1 transmits a short signal, on the other hand, a node whose $\mathrm{CN}$ value is 0 keeps silent and senses channel whether there is any signal. If it finds the slot is busy, it loses the competition; otherwise, it survives and remains in the competition. So only the node that survives all the bit-slots is allowed to transmit control messages. However, the mechanism assumes all competing nodes are synchronized and start transmitting $\mathrm{CN}$ signal in the same slot. It is hard to realize in the distributed networks.

In order to meet the demand of efficient medium accessing in multi-hop ad hoc networks, we propose a QoS-based multiple access (QMA) protocol, which implements forecast bursts (FB) with priorities to achieve random contention access, 
collision resolution and the QoS guarantee for different traffic. The rest of this paper is organized as follows. In section 2 , we provide the network model. The QMA protocol is presented in section 3. Section 4 discusses simulations and the results. We conclude the paper in section 5 .

\section{Network Model}

The QMA protocol is based on carrier sensing networks. In [8], it states the range covered by the power necessary for transmitting a packet from a transmitter has two disjoint areas, named transmission range and carrier sensing zone. Within the transmission range, a node can sense and decode a signal correctly, whereas a node can sense but can not decode it correctly in the carrier sensing zone. Usually the carrier sensing range is more than twice of the transmission range. Therefore, a node can overhear the channel within its 2-hop transmission range by judging radio signal strength, in another word, the node in its MAC layer can judge whether the other nodes within its 2-hop range are transmitting or not to avoid a possible collision. As a result, the hidden terminal problem is solved in the QMA protocol.

\section{Proposed QMA Protocol}

In the QMA protocol, channel access cycle is divided into contention phase and transmission phase, and a node must sense the medium for a time interval $\mathrm{T}_{\text {win }}$ before accessing. If the channel is idle, the node is allowed to start contention phase; otherwise, it defers to wait for another attempt. The contention phase consists of $(\mathrm{n}+\mathrm{m})$ mini slots, each $\mathrm{T}_{\text {slot }}\left(\mathrm{T}_{\text {slot }}<\mathrm{T}_{\text {win }}\right)$ long. Among them the former $\mathrm{n}$ slots are assigned to the nodes with real-time traffic to contend by broadcasting $\mathrm{FBs}\left(\mathrm{T}_{\mathrm{FB}}=\mathrm{T}_{\mathrm{slot}}\right)$, and the latter $\mathrm{m}$ slots are assigned to the nodes with non real-time traffic. Therefore, the nodes with non real-time traffic are allowed to broadcast FBs only on condition that all the former $\mathrm{n}$ slots for real-time traffic are idle. In this way, the requirements of real-time traffic are guaranteed prior to that of non real-time traffic. If a node senses the first B slots idle, it will start broadcasting k.FBs from the Bth slot in this contention phase. Otherwise, it stops contending and performs back-off. B is a random variable with a truncated geometric distribution. For the nodes that have realtime traffic, B is a random variable as follows:

$$
\operatorname{Pr}(B=b)=\left\{\begin{array}{cc}
(1-q) q^{b} & 0 \leq b<n \\
q^{n} & b=n
\end{array}\right.
$$

For the nodes that have non real-time traffic, B is a random variable as follows:

$$
\operatorname{Pr}(B=b)=\left\{\begin{array}{cc}
(1-q) q^{b} & n \leq b<n+m \\
q^{m} & b=n+m
\end{array}\right.
$$

In which, $\mathrm{q}$ is decided by the category and delay of the packet. The $\mathrm{k}$ is related to delay of packets according to the EDF principle, i.e., $\mathrm{k}$ is adjusted dynamically and the packet with earliest deadline has the largest $\mathrm{k}$. 
After broadcasting FBs, the node will sense the channel for the duration of $\mathrm{T}_{\mathrm{obs}}$ $\left(\mathrm{T}_{\mathrm{obs}}=\mathrm{T}_{\text {slot }}\right)$. If the channel is sensed as busy during the $\mathrm{T}_{\mathrm{obs}}$ interval, there must be at least one node with higher priority packet trying to access, so that the node has to perform back-off. Otherwise, if the channel is sensed as being idle, the node will win the contention and start transmission in the next transmission phase. The intended receiver will reply an acknowledgement (ACK) packet after receiving the packet correctly. As shown in Fig. 1, node A and B start broadcasting FBs in the same slot in the contention phase, while node $\mathrm{C}$ intends to start broadcasting FBs in the latter slot according to the priority and delay of its packet. Hence the node $\mathrm{C}$ senses the channel busy before it broadcasts FBs, and it stops contending and performs back-off. For the other two nodes, node A broadcasts more FBs than node B; therefore node A wins the contention and sends a packet in the transmission phase, while node $\mathrm{B}$ also performs back-off.

The above discussion illustrates that the nodes are allowed to broadcast FBs on condition that the channel is sensed as idle during first B slots in contention phase. This algorithm guarantees only the nodes which start broadcasting FBs in the same slot can become candidates in contention phase, and the node that broadcasts the most FB packets wins the contention and accesses the channel successfully. Since a packet with the highest priority and the earliest deadline results in the most FBs, the node with the packet will get to access the channel and transmit the packet at once.

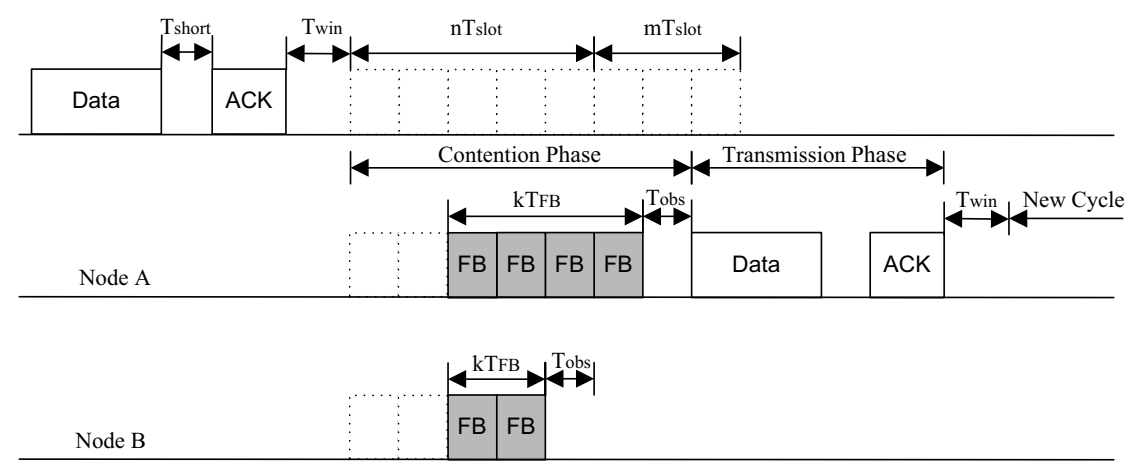

Fig. 1. An Example of QMA Protocol

\section{Performance Evaluation}

\subsection{Simulation Environment}

To evaluate the effectiveness of QMA's QoS support, we compare the performance of QMA protocol with CSMA/CA protocol using the OPNET modeler. The simulation 
has modeled a network of $\mathrm{N}$ nodes randomly placed in an area named as $\mathrm{A}$. The transmission radius is $\mathrm{R}$, and hence, the receivers can sense the channel in a 2-hop transmission range (i.e., 2R). Assume that message generation process follows Poisson process with average arrival rate $\lambda$, and the number of data packets (PKT) per message follows truncated geometric distribution with the maximum number $\mathrm{L}_{\mathrm{M}}$ and probability parameter $q_{\mathrm{PKT}}$. The retransmission limit is $\mathrm{L}$. Respectively, $\mathrm{L}_{\mathrm{FB}}$ and $\mathrm{L}_{\mathrm{PKT}}$ are the lengths of FB packets and data packets. The parameter $\mathrm{q}$ of random variable $\mathrm{B}$ is a constant for simplicity in simulations. The number of FB packets is $k(0<k \leq n)$, $\mathrm{k} \propto \mathrm{D} / \mathrm{D}_{\max }$, in which $\mathrm{D}_{\max }$ is the lifetime of the packet. The detailed parameters in QMA protocol simulations are set in Table. 1. Meanwhile, the simulation of CSMA/CA protocol is set in the same scenario with 40 nodes. For a good performance, the lengths of data packet and ACK packet in CSMA/CA are set to 4 kbits and 40 bits, and sensing interval is $0.1 \mathrm{~ms}$.

Table 1. Simulation Parameters of QMA Protocol

\begin{tabular}{|c|c|c|c|c|c|c|c|c|c|c|c|c|}
\hline \multicolumn{2}{|c|}{ Scenario } & Channel & \multicolumn{2}{c|}{ Message } & \multicolumn{4}{c|}{ Intervals } & \multicolumn{3}{c|}{ Packet } \\
\hline $\begin{array}{c}\mathrm{A} \\
/ \mathrm{km}^{2}\end{array}$ & $\begin{array}{c}\mathrm{R} \\
/ \mathrm{km}\end{array}$ & $\begin{array}{c}\text { Data rate } \\
/(\mathrm{Mb} / \mathrm{s})\end{array}$ & $\mathrm{L}_{\mathrm{M}}$ & $\mathrm{q}_{\mathrm{PKT}}$ & $\mathrm{m}$ & $\mathrm{n}$ & $\begin{array}{c}\mathrm{T}_{\text {win }} \\
/ \mathrm{m}\end{array}$ & $\begin{array}{c}\mathrm{T}_{\text {slot }} \\
/ \mathrm{us}\end{array}$ & $\mathrm{q}$ & $\begin{array}{c}\mathrm{L}_{\mathrm{FB}} \\
/ \mathrm{bit}\end{array}$ & $\begin{array}{c}\mathrm{L}_{\mathrm{PKT}} \\
/ \mathrm{bit}\end{array}$ & $\mathrm{L}$ \\
\hline $50 \times 50$ & 10 & 2 & 40 & 0.95 & 12 & 12 & 268 & 64 & 0.8 & 128 & 18500 & 7 \\
\hline
\end{tabular}

\subsection{Observed Simulation Results}

Normalized channel throughput (normalized by average hops between nodes for the concurrent transmissions in multi-hop networks), average message discard rate and average per hop packet delay of QMA protocol and CSMA/CA protocol are shown in the figure 2, 3 and 4 .

As seen from Fig. 2, the saturated throughput of QMA is higher than that of CSMA/CA protocol. Before $\lambda<1$, the throughput of QMA protocol increases lineally with the increase of $\lambda$; after $\lambda>1$ especially after $\lambda>1.5$, that increases slowly for the load is reaching to the limit of transmission capacity and packets might experience more contention cycles or retransmissions. And as the number of nodes increasing in simulations, the saturated throughput rises a little for there might be more concurrent transmissions. The results illustrate that the performance also depends on node density in multi-hop networks.

In the Fig.3, the CSMA/CA protocol which uses shorter data packet has a lower average message discard rate than QMA protocol when offered load is low. With the increase of offered load, nodes in both protocols suffer from more collisions and more dropped messages due to overtime or the limit of retransmission times. Compared with CSMA/CA protocol, the QMA protocol has a better performance when offered load is high, because the contention of FB guarantees that only one node among many competitors can successfully get to access and transmit its packet without collision.

The Fig. 4 shows average per hop packet delay of real-time and non real-time traffic with different nodes. The CSMA/CA protocol has a better performance when 


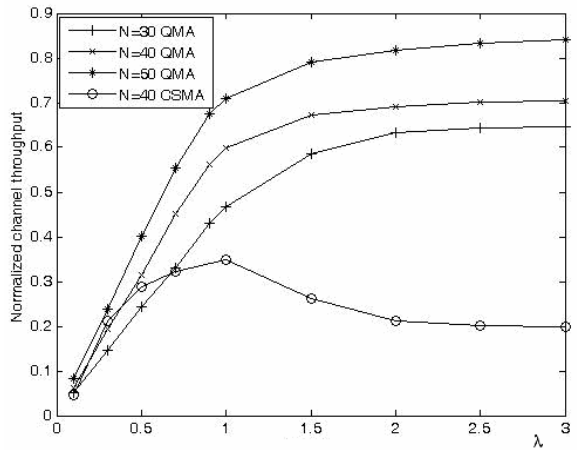

Fig. 2. Normalized Channel Throughput

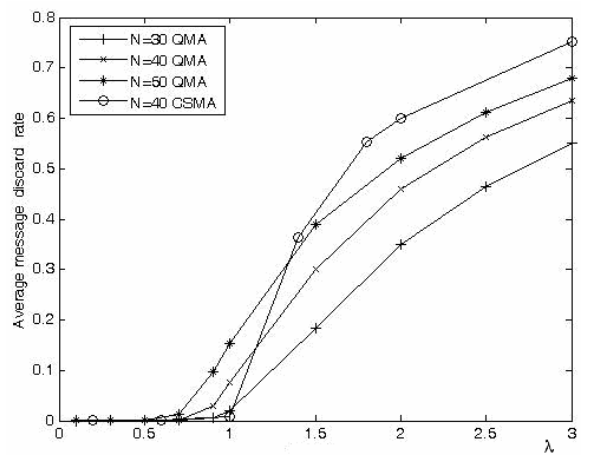

Fig. 3. Average Message Discard Rate

$\lambda<1$, because nodes can send packets after sensing a short interval. However, the increase of collisions and back-off time deteriorates average packet delay drastically when offered loads become larger. In QMA protocol, even though the delay increases with load increasing, the delay of real-time packets is guaranteed and bounded in $1 \mathrm{~s}$ because the real-time packets access prior to non real-time packets in contentions. Before offered load reaching to the transmission capacity, the average delay of realtime packets remains very low while the delay of non real-time packets is increasing. In another word, the QMA protocol guarantees QoS of real-time packets with the price of the delay of non real-time packets.

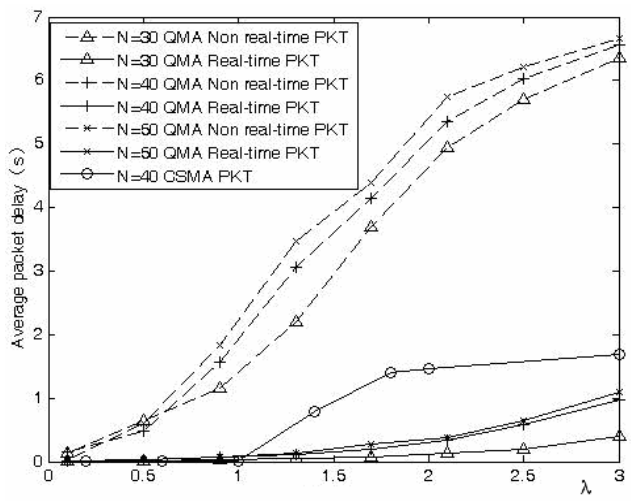

Fig. 4. Average per Hop Packet Delay

\section{Conclusions}

In this paper, the QMA protocol supporting multimedia service and multi-hop architecture is proposed for mobile ad hoc networks, which integrates the concepts of contention reservation and collision resolution. The nodes contend to access the channel by broadcasting FBs before packet transmission. Therefore, the QMA 
protocol can decrease the possibility of data packet collision and support QoS guarantee for the real-time traffic. The simulation results have shown that the QMA protocol can provide effective channel sharing for nodes compared with CSMA/CA protocol, and guarantee QoS requirements for different traffic.

Acknowledgements. This work was supported by the National Nature Science Foundation of China (No.10577005 and No.60625102), the National Defense PreResearch Project (No.513250403), and the Innovation Foundation of Aerospace Science and Technology of China.

\section{References}

1. IEEE Standard for Wireless LAN Medium Access Control (MAC) and Physical Layer (PHY) Specifications (1999)

2. Chen, Y., Zeng, Q.A., Agrawal, D.P.: Performance analysis and enhancement for IEEE 802.11 MAC protocol. In: Proc. IEEE ICT 2003 vol. 4, pp. 860-867 (2003)

3. Xu, S., Saadawi, T.: Does the IEEE802.11 MAC protocol work well in multihop wireless ad hoc networks? IEEE Commun. Mag. 39(6), 130-137 (2001)

4. Yun, L.: D-PRMA: A Novel MAC Mechanism Supporting Resource Reservation for Wireless Ad Hoc Networks. Journal of Electronics \& Information Technology 27(6), 967-972 (2005)

5. Hayoung, Y., JongWon, K., DongYun, S.: Dynamic Admission Control in IEEE 802.11e EDCA-based Wireless Home Network. In: Consumer Communications and Networking Conference, vol. V1, pp. 55-59 (2006)

6. Xiao, Y., Pan, Y.: Differentiation, QoS Guarantee, and Optimization for Real-Time Traffic over One-Hop Ad Hoc Networks. IEEE Transactions on Parallel and Distributed Systems 16(6), 538-549 (2005)

7. Yaling, Y., Kravets, R.: Distributed QoS guarantees for realtime traffic in ad hoc networks. In: Sensor and Ad Hoc Communications and Networks (IEEE SECON), October 2004, pp. 118-127 (2004)

8. Choi, W.C., Lee, Y.S., Rhee, S.H., et al.: End-to-end performance improvements for multi-hop ad-hoc wireless networks. In: Software Technologies for Future Embedded and Ubiquitous Systems, May 2004, pp. 53-57 (2004)

9. Anastasi, G., Lenzini, L., Mingozzi, E.: Stability and Performance Analysis of HIPERLAN. In: IEEE INFOCOM '98, San Francisco, CA, vol. 1, pp. 134-141 (1998)

10. Yeh, C.H., You, T.A.: A QoS MAC protocol for differentiated service in mobile ad hoc networks. In: The 2003 International Conference on Parallel Processing (ICPP'03), pp. 349-356 (2003) 